11. Kaushik KS, Lahiri KK, Chumber SK, et al. Molecular characterization of clinical varicella-zoster strains from India and differentiation from the oka vaccine strain. Japanese Journal of Infectious Diseases 2008; 61: 65-7.

12. Sharrar RG, LaRussa P, Galea SA, et al. The postmarketing safety profile of varicella vaccine. Vaccine 2000; 19: 916-23.

13. Quinlivan ML, Gershon AA, Al Bassam MM, et al. Natural selection for rash-forming genotypes of the varicella-zoster vaccine virus detected within immunized human hosts. Proceeding of the National Academy of Sciences of the United States of America 2007; 104: 208-12.
14. Salzman MB, Sharrar RG, Steinberg S, LaRussa P. Transmission of varicella-vaccine virus from a healthy 12 month-old child to his pregnant mother. The Journal of Pediatrics 1997; 131: 151-4.

15. Grossberg R, Harpaz R, Rubtcova E, et al. Secondary transmission of varicella vaccine virus in a chronic care facility for children. The Journal of Pediatrics 2006; 148: 842-4.

16. LaRussa P, Steinberg S, Meurice F, Gershon A. Transmission of vaccine strain varicella-zoster virus from a healthy adult with vaccine-associated rash to susceptible household contacts. The Journal of Infectious Diseases 1997; 176: $1072-5$.

\title{
Validation of the Sinhala version of the Chronic Liver Disease Questionnaire (CLDQ) for assessment of health related quality of life among Sri Lankan cirrhotics
}

\author{
C K Ranawaka ${ }^{1}$, J K N D Miththinda ${ }^{1}$, S M Senanayake ${ }^{1}$, W R S de Alwis ${ }^{2}$, M N F Mufeena ${ }^{2}$, M A \\ Niriella $^{2}$, A S Dassanayake ${ }^{3}$, A P de Silva ${ }^{2}$, A Pathmeswaran ${ }^{4}$, H J de Silva ${ }^{2}$ \\ (Index words: cirrhosis, quality of life (QOL), Chronic Liver Disease Questionnaire (CLDQ))
}

\begin{abstract}
Objectives The Chronic Liver Disease Questionnaire (CLDQ) is a validated tool measuring Health Related Quality of Life among patients with cirrhosis. The aim of this study was to validate a Sinhala version of the CLDQ (SCLDQ) and to test its correlation with the degree of liver dysfunction in a cohort of Sri Lankan patients with cirrhosis.

Methods A standard translation method was used. Pilot testing was done with relevant cultural and language adaptations. The final version and the WHO Quality of Life-BREF (WHOQOL-BREF) validated Sinhala version were administered to patients with chronic lever disease (CLD). SCLDQ was re-administered 4 weeks later to test internal consistency and reliability. The validaty and reliability were assessed by Cronabach's alpha, intraclass correlation coefficient (ICC) and Pearson's correlation coefficient. ANOVA and Pearson's correlation were used to assess correlation with the degree of liver dysfunction.
\end{abstract}

Results Validation was done with 214 participants [mean age 55.6 years (SD 10.4) male 77.6\%]. Cronabach's alpha was 0.926 . Intra-class correlations varied from
0.431 to 0.912 and all were significant $(p<0.001)$. Retesting was done on a sub-sample of 18 participants. Test-retest correlation was $0.695(p=0.008)$. WHO-BREF was administered to a sub-sample of 48 subjects. There was a significant correlation (Pearson's $r=0.391$; $p=0.004$ ) between SCLDQ and WHOQOL BREF. SCLDQ was significantly associated with MELD $(r=-0.13$; $p=0.038)$, MELD sodium $(r=-0.223 ; p=0.002)$, serum bilirubin ( $r=-0.124 ; p=0.036)$, serum sodium $(r=0.172$; $p=0.009)$, serum albumin $(r=0.201 ; p=0.003)$ and Child grade $(\mathrm{f}=3.687 ; p=0.027)$.

Conclusions CLDQ is a reliable and valid tool to assess quality of life of Sri Lankan patients with cirrhosis and correlates well with known indices of disease severity.

Ceylon Medical Journal 2013; 58: 156-62

\section{Introduction}

Health related quality of life (HRQL) is important in people with chronic diseases. Cirrhosis of the liver has a considerable effect on patients' physical and psychosocial wellbeing. The progression of symptoms, functional

${ }^{1}$ University Medical Unit, Colombo North Teaching Hospital, and Departments of ${ }^{2}$ Medicine, ${ }^{3}$ Pharmacology and ${ }^{4}$ Public Health, Faculty of Medicine, University of Kelaniya, Ragama, Sri Lanka.

Correspondence: JKNDM, e-mail: <jkndmiththinda@gmail.com>. Received 4 June 2013 and revised version accepted 9 October 2013. Competing interests: none declared. 
limitation associated with increasing severity of liver disease and complications such as hepatic encephalopathy, ascites, spontaneous bacterial peritonitis, and recurrent variceal haemorrhage may lead to repeated health visits and hospitalisation leading to financial problems and poor quality of life (QOL).

Several disease-specific symptoms such as pruritus, muscle cramps, sleep disturbance, sexual dysfunction, fatigue, and gastrointestinal symptoms have an effect on the QOL in patients with cirrhosis [1]. Knowledge of symptom profiles in chronic liver disease (CLD) may help in the development of more comprehensive treatment plans. Child-Turcotte-Pugh (CTP), model for end stage liver disease (MELD) and MELD-sodium (MELD-Na) are well known scoring systems of disease severity that depend on laboratory investigations, but do not measure QOL [2-4]. Assessment of HRQL is important for these patients in view of the paucity of therapies except for liver transplantation that substantially improve survival. Liver transplantation improves QOL but it is not yet wellestablished in Sri Lanka [5-8]. Even in countries where it is available, there is a significant mismatch between demand and supply of organs.

The Chronic Liver Disease Questionnaire (CLDQ) developed by Younossi et al is the first liver specific questionnaire, designed to measure HRQL [9]. CLDQ is short and easy to administer, and is designed to measure six domains of QOL; abdominal symptoms, fatigue, systemic symptoms, activity, emotional functions and worry. All 29 items assess subjective symptoms over the previous 2 weeks on a 7 point Likert scale, with 1 corresponding to the maximum frequency ("all of the time") and 7 to the minimum ("none of the time"). Domain scores are derived from the means of the items in each domain and the overall score is the mean of all domains.

CLDQ has been translated into different languages around the world and validated. It has shown good correlation with severity of liver disease [10-15]. Despite the growing health burden associated with cirrhosis in Sri Lanka, there have been no attempts to evaluate QOL of patients with chromic liver disease (CLD). The aim of this study was to validate a Sinhala version of the CLDQ and to test its correlation with the degree of liver dysfunction in a cohort of Sri Lankan patients with cirrhosis.

\section{Methods}

This study was carried out in the Gastroenterology Clinic, University Medical Unit, Colombo North Teaching Hospital, Ragama. The study was carried out in two steps: translation of the Sinhala version of CLDQ (sCLDQ) and validation of the translated SCLDQ.

\section{Translation of CLDQ to Sinhala}

A standard method of translation introduced by the MAPI Research Institute was used for the translation of
CLDQ to Sinhala (sCLDQ) $[16,17]$. Definition of concepts of each item of the CLDQ and forward translation was carried out by two bilingual translators. A meeting was held to agree on the final version, and back translation of this version was performed by a third translator. After comparison of the original and the back translation by the interim investigators, pilot testing was carried out on 10 patients with stable CLD. Relevant adaptations were done according to differences in culture and language. The final translated version had optimal both linguistic and conceptual equivalence.

\section{Validation of the SCLDQ}

A cross sectional study was carried out to validate the sCLDQ in the outpatient clinic over a three month period. Patients with cirrhosis who spoke Sinhala and attending follow up clinics from December 2011 were recruited after obtaining informed written consent. Patients with clinically stable CLD of any aetiology between 18 and 70 years of age were included. Patients with other uncontrolled co-morbidities such as congestive heart failure, diabetes, hypertension with complications, end stage chronic kidney disease, stroke, active inflammatory bowel disease or malignancies were excluded. Patients who had hepatic encephalopathy grade 2 or worse, a hospital admission within the past two weeks, active psychological ailments or those who were illiterate were also excluded.

Of the 628 patients with cirrhosis in the data base, 214 were recruited for the final study. Relevant demographic, socioeconomic, clinical and biochemical information was obtained using an interviewer administered data extraction form. sCLDQ was self-administered. Of the 214 participants, WHOQOL-BREF was administered to 48 [18]. Following a period of 4 weeks, re-testing was done on 18 participants. WHOQOL-BREF contains four domains of QOL: physical, psychological, social and environments. It has been translated into several languages and validated. It has been used to assess QOL in a large number of different diseases around the world. In Sri Lanka, WHOQOL-BREF was validated in a cohort of patients with polycystic ovarian disease [19].

\section{Data Analysis}

Data were analysed using SPSS statistical package version 21. Demographic and clinical characteristics were described for the total sample, WHOQOL-BREFF subsample and re-test sub-sample. Distribution of scores was described for the total score and domain scores by calculating mean, standard deviation, proportion of patients with a missing item, range, percentage of patients with worst (floor) and best (ceiling) scores.

Reliability was assessed using internal consistency and reproducibility. Internal consistency was evaluated with Cronbach's alpha co-efficient. Reproducibility was 
evaluated with intra class correlation and test-retest scores. Intra-class correlation coefficient was calculated with a two-way-mixed and absolute agreement models. Test-retest correlation was assessed with Pearson's correlation co-efficient. A correlation co-efficient of 0 conformed to no agreement at all and 1 to full agreement.

Structural validity, the extent to which placement of items into domains agreed with the distribution of the original questionnaire was assessed using exploratory factor analysis with varimax rotation. Number of factors was determined with Monte-Carlo parallel analysis.

Construct validity, the extent to which SCLDQ scores demonstrated logical relations with other health related quality of life and clinical measures was assessed with correlation of sCLDQ score with WHOQOL- BREF score, MELD score, platelet count, serum sodium, bilirubin and albumin levels. Relationship with Child grade was assessed with one-way analysis of variance (ANOVA) and post hoc analysis was done using Tukey's test.

Approval to conduct the study was obtained from the Ethics Committee of the Faculty of Medicine, University of Kelaniya.

\section{Results}

A total of 214 patients with chronic liver disease [mean age 55.6 years (SD 10.4), 77.6\% males] were recruited to validate the questionnaire. All were patients with cirrhosis either without any co-morbidities or with stable co-morbidities. Nearly half the patients had alcohol cirrhosis (Table 1). The average duration of CLD was 2.5 years (SD 2.8) and most were Child A grade. From this total cohort, the WHOQOL-BREF questionnaire was administered to a sub-sample of 48 participants and a retest was carried out in 18 subjects. These sub-samples had an age-sex distribution corresponding with the total cohort. However, the majority of the WHOQOL-BREF sub-sample comprised of Child B grade. Cryptogenic/NASH cirrhosis was commoner in both sub-samples compared to the total cohort (Table 1).

The total and domain score distributions for SCLDQ are summarized in Table 2. The mean item scores ranged from 3.8 to 5.3. Only $35(16.4 \%)$ patients needed assistance in completing the questionnaire. The questionnaire had good understandability with only 3 missing items (1.14\%). Overall and domain scores ranged from 1 to 7 . The overall floor and ceiling effects were minimal except for the domains of 'abdominal symptoms' and 'worry' which showed higher ceiling effects with 30 (14\%) and 31 (14.6\%) respectively. sCLDQ had an overall Cronbach's alpha of 0.926 and 4 out of 6 domains had cronbach's alpha values over 0.7. Overall intra-class correlation was $0.912(p<0.001)$ and test-retest correlation was $0.695(p=0.008)$. 'Systemic symptoms' and 'activity', the two domains with lower alpha values (0.607 and 0.484$)$, showed significant intra-class correlation ( $\mathrm{r}=0.552$ and 0.431 respectively, $p=0.000)$ and test-retest correlations $(\mathrm{r}=0.755$ and 0.784 respectively, $p=0.001)$. Though 'fatigue' didn't show a significant test-retest correlation $(\mathrm{r}=0.430, p=0.125)$ the Cronbach's alpha was 0.813 . It had significant intra-class

Table1. Demographic and clinical characteristics

\begin{tabular}{lccc}
\hline \multicolumn{1}{c}{ Characteristic } & $\begin{array}{c}\text { Total sample } \\
n=214\end{array}$ & $\begin{array}{c}\text { WHOQOL-BREF } \\
\text { Sub sample } n=48\end{array}$ & $\begin{array}{c}\text { Re-test sub sample } \\
n=18\end{array}$ \\
\hline Age, mean(SD) years & $55.6(10.4)$ & $55.6(10.0)$ & $56.4(10.1)$ \\
Gender (males), n (\%) & $166(77.6 \%)$ & $38(79.2)$ & $12(80 \%)$ \\
Severity, n (\%) & $124(57.9 \%)$ & $17(35.4 \%)$ & $8(44.4 \%)$ \\
Child A & $59(27.6 \%)$ & $20(41.7 \%)$ & $5(27.8 \%)$ \\
Child B & $31(14.5 \%)$ & $11(22.9 \%)$ & $5(27.8 \%)$ \\
Child C & & $18(37.5 \%)$ & $5(27.8 \%)$ \\
Aetiology, n (\%) & $105(49.1 \%)$ & $28(58.3 \%)$ & $13(72.2 \%)$ \\
Alcohol & $93(43.5 \%)$ & $2(4.2 \%)$ & $0(0.0 \%)$ \\
Cryptogenic & $16(7.4 \%)$ & $17(35.4 \%)$ & $9(50 \%)$ \\
Other & $106(49.5 \%)$ & $17(35.4 \%)$ & $4(22.2 \%)$ \\
Co-morbidities, n (\%) & $63(29.4 \%)$ & $5(10.4 \%)$ & $3(16.7 \%)$ \\
None & $21(9.8 \%)$ & $3(6.3 \%)$ & $0(0.0 \%)$ \\
Diabetes only & $7(3.3 \%)$ & $1(2.1 \%)$ & $0(0.0 \%)$ \\
Diabetes and other & $3(1.4 \%)$ & $5(10.4 \%)$ & $2(11.1 \%)$ \\
Hypertension only & $14(6.5 \%)$ & & \\
Hypertension and other & & & \\
Others & & & \\
\hline
\end{tabular}


correlation $(r=0.808, p=0.000)$. Monte-Carlo parallel analysis showed 8 domains as opposed to the 6 domains described in the original questionnaire. Exploratory factor analysis according to these eight domains is described in Table 3. Items 16 and 20 relating to disturbance of sleep and items 4 and 13 relating to drowsiness / sleepiness appeared as new domains. The original domains retained most of their items; abdominal symptoms (2/3), fatigue $(3 / 5)$, systemic symptoms $(2 / 5)$, activity $(2 / 3)$, emotional function (6/8) and worry (5/5).

Sub-sample analysis of the WHOQOL-BREF questionnaire showed a small but significant positive correlation between SCLDQ scores and WHOQOL-BREF domains. Total and all sub-domain scores of sCLDQ except abdominal symptoms correlated significantly with "physical" and "psychological" sub-domains of WHOBREF $(r>0.2, p<0.05)$. sCLDQ “Abdominal symptoms" correlated with the physical sub-domain of WHO BREF (r 0.297, $p=0.020$ ). "Social" and "environmental" subdomains of WHO-BREF did not correlate significantly with total or sub-domain scores of sCLDQ.

sCLDQ had a significant positive correlation with serum sodium $(\mathrm{r}=0.172, p=0.009)$ and albumin levels $(\mathrm{r}=0.201, p=0.003)$, and a negative correlation with MELD score $(\mathrm{r}=-0.130, \mathrm{p}=0.038)$, MELD sodium score $(\mathrm{r}=-0.223$, $p=0.002)$ and serum bilirubin $(\mathrm{r}=-0.124, p=0.036)$. It did not have a significant correlation with ascites $(p=0.458)$, platelet count $(\mathrm{r}=0.008, p=0.455), \mathrm{INR}(\mathrm{r}=0.018, p=0.400)$ and serum creatinine $(\mathrm{r}=-0.062, p=0.202)$. sCLDQ total score varied significantly according to the Child grade $(\mathrm{f}=3.687, p=0.027)$. Post-hoc analysis showed significant diference between Child grade B and C ( $p=0.023)$. None of the individual domains showed a significant variation between Child grades (Figure 1).

Table 2. Distribution of sCLDQ scores

\begin{tabular}{|c|c|c|c|c|c|c|}
\hline Scales & Mean & $S D$ & Missing & Observed range & Floor (\%) & Ceiling (\%) \\
\hline Total & 4.5 & 1.0 & 3 & $2.3-6.9$ & 0.5 & 0.5 \\
\hline Abdominal symptoms & 5.3 & 1.4 & 0 & $1.3-7$ & 0.9 & 14 \\
\hline Fatigue & 3.8 & 1.2 & 1 & $1-7$ & 0.9 & 0.5 \\
\hline Systemic symptoms & 4.6 & 1.1 & 0 & $1.8-7$ & 0.5 & 1.4 \\
\hline Activity & 4.6 & 1.3 & 1 & $1-7$ & 0.5 & 6.6 \\
\hline Emotional function & 4.4 & 1.3 & 0 & $1.5-7$ & 0.9 & 3.7 \\
\hline Worry & 4.9 & 1.5 & 1 & $1.4-7$ & 0.9 & 14.6 \\
\hline
\end{tabular}

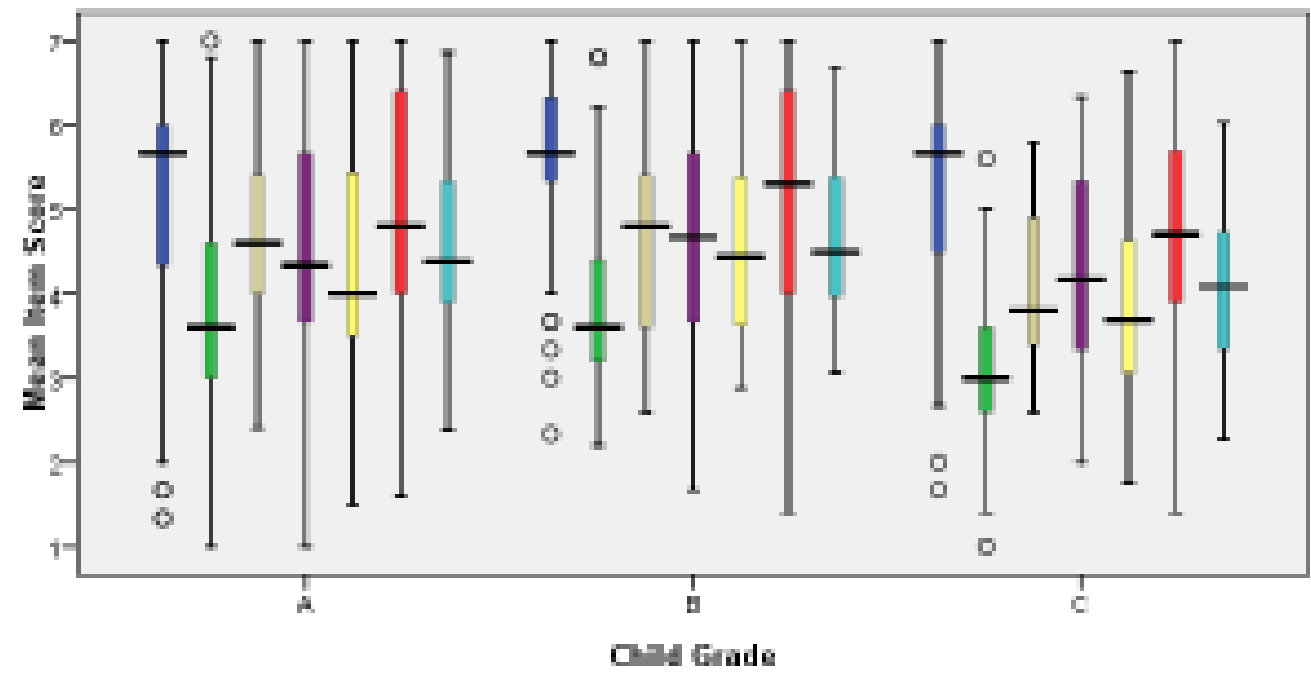

Figure 1. Variation of SCLDQ score items by Child Grade. 
Table 3. Exploratory factor analysis loading values

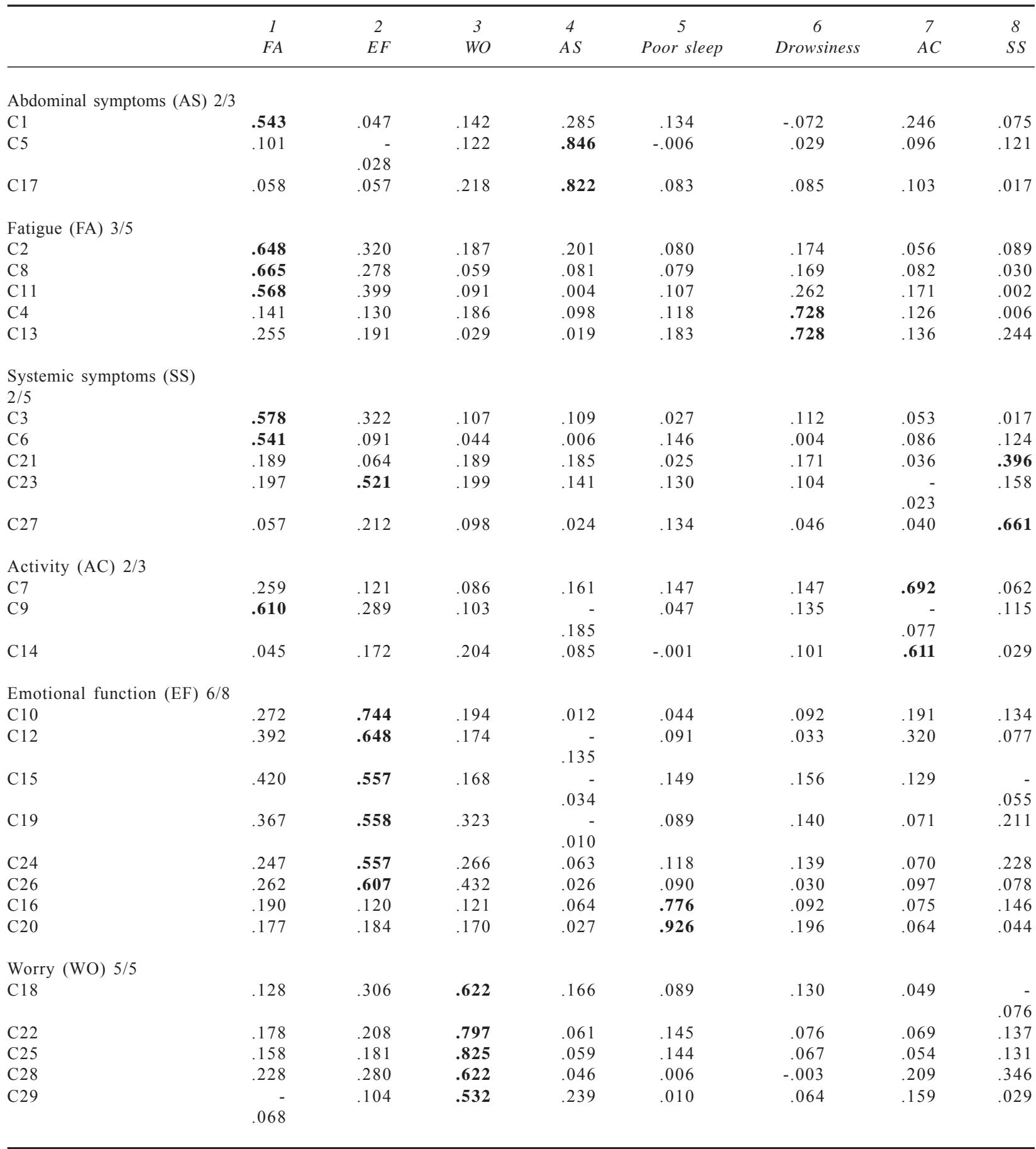

\section{Discussion}

The Sinhala version of CLDQ could be easily selfadministered with minimal assistance and was well accepted by patients. The total and domain score distributions were similar to the original questionnaire and other translated versions. We found the SCLDQ to be a reliable and valid tool to assess QOL of Sinhala speaking Sri Lankan patients with cirrhosis. It correlated well with known indices of disease severity.

The overall internal consistency was excellent and, except the 'activity' domain, the domains showed moderate to high internal consistencies. Poor internal consistency of the 'activity' domain was largely due to the 9th Item: 'How often during the last two weeks have you had trouble lifting or carrying heavy objects?' This may have been due to cultural differences between the original study cohort and our cohort, where adoption of a sick-role is commoner. Also, what is considered 'heavy' may differ between the cohorts. Though the removal of this item increased the internal consistency of the 'activity' domain, it had a minimal effect on further 
improving the overall internal consistency; therefore it was decided to keep this item in the final sCLDQ.

Pilot testing of the sCLDQ identified some inconsistencies. There was no difference in responses for items 4 and 13 (How often during the last two weeks have you felt sleepy during the day? and How often during the last two weeks have you felt drowsy?) as well as between items 5 and 17 (How much of the time during the last two weeks have you experienced abdominal pain? and How much of the time during the last two weeks have you been troubled by a feeling of abdominal discomfort?). This could be due to lack of exact language constructs to differentiate these entities from each other.

The original CLDQ was re-tested after six months but we carried out the re-test after four weeks. A period of 4 weeks was selected as it was considered the best time duration to balance the inconsistencies due to progression of the disease and recall bias. Test-retest correlation showed moderate to high significant correlations, except in the 'fatigue' domain. This is probably due to items 4 and 13 relating to drowsiness, the removal of which increased the test-retest correlation of this domain significantly. This is further supported by identification of, 'drowsiness' as a separate domain. Poor test-retest correlation may be due to variability of a symptom like drowsiness over a period of 4 weeks. Despite these minor inconsistencies in test-retest correlations, overall and individual-domain intra-class correlations remained highly significant, thereby confirming the reliability of SCLDQ.

The original 6 domain structure of CLDQ was retained in the Sinhala version, with two additional domains. The 'worry' domain retained all of its items as in the original CLDQ. Items related to sleep disturbance (Items 16 and 20) which were listed under the 'emotional function' domain in the original CLDQ, appeared as a separate domain (poor sleep domain) while the rest of the elements of that domain remained together. Two out of three elements of the original 'activity' domain remained together, while item 9 loaded on the 'fatigue' domain. The elements which remained in the 'activity' domain were related to the diet, while item 9 , regarding carrying heavy objects was considered a 'fatigable' activity rather than a simple day to day activity by to our cohort. Items 4 and 13 of the 'fatigue' domain appeared as a separate 'drowsiness' domain. In the 'systemic symptoms' domain, element 3 regarding bodily pain and element 6 regarding shortness of breath, correlated more with the 'fatigue' domain. This could probably be due to linguistic issues where the differentiation between these terms is blurred.

sCLDQ total showed significant positive correlation with the physical and psychological domains of the WHOQOL-BREF questionnaire. It did not show significant correlation with social and environmental domains which may be because those aspects are only minimally addressed by the CLDQ. sCLDQ showed low but signi- ficant correlation with MELD score, MELD sodium score, serum sodium, albumin and bilirubin levels reflecting its construct validity. The significant difference of sCLDQ scores between Child B and C categories further con-firmed this.

In conclusion, even though there are some limitations, the Sinhala version of the CLDQ is a valid and reliable tool to assess the quality of life of Sinhala speaking patients with cirrhosis. This is important given the growing health burden associated with cirrhosis in Sri Lanka.

\section{References}

1. Kalaitzakis E. Quality of Life in Liver Cirrhosis. In: Preedy VR, Watson RR, eds. Handbook of Disease Burdens and Quality of Life Measures: New York 2010: 2239-54.

2. Child CG, Turcotte JG. Surgery and portal hypertension. Major Problems in Clinical Surgery 1964; 1: 1-85.

3. Kamath PS, Wiesner RH, Malinchoc M, et al. A model to predict survival in patients with end-stage liver disease. Hepatology 2001; 33: 464-70.

4. RufAE, Kremers WK, Chavez LL, et al. Addition of serum sodium into the MELD score predicts waiting list mortality better than MELD alone. Liver Transplantation 2005; 11: $336-43$.

5. Leyendecker B, Bartholomew U, Neuhaus R, et al. Quality of Life of Liver Transplant Recipients: A Pilot Study. Transplantation 1993; 56: 561-7.

6. Hicks FD, Larson JL, Ferrans CE. Quality of Life after Liver Transplant. Research in Nursing and Health 1992; 15: 111-9.

7. Duffy JP, Kao K, Ko CY, et al. Long-Term Patient Outcome and Quality of Life After Liver Transplantation: Analysis of 20-Year Survivors. Annals of Surgery 2010; 252: 652-61.

8. Ruppert K, Kuo S, DiMartini A, Balan V. In a 12-Year Study, Sustainability of Quality of Life Benefits After Liver Transplantation Varies With Pretransplantation Diagnosis. Gastroenterology 2010; 139: 1619-29. e4.

9. Younossi ZM, Guyatt G, Kiwi M, Boparai N, King D. Development of a disease specific questionnaire to measure health related quality of life in patients with chronic liver disease. Gut.1999; 45: 295-300.

10. Ray I, Dutta D, Basu P, De BK. Quality of life assessment of patients with chronic liver disease in eastern India using a Bengali translation chronic liver disease questionnaire. Indian Journal of Gastroenterolgy 2010; 29: 187-95.

11. Atiq M, Gill ML, Khokhar N. Quality of life assessment in Pakistani patients with chronic liver disease. Journal of Pakistan Medical Association 2004; 54: 113-5.

12. Sobhonslidsuk A, Silpakit C, Kongsakon R, Satitpornkul P, Sripetch C. Chronic liver disease questionnaire: translation and validation in Thais. World Journal of Gastroenterolgy 2004; 10: 1954-7.

13. Rucci P, Taliani G, Cirrincione L, Alberti A, Bartolozzi D, Caporaso $\mathrm{N}$, et al. Validity and reliability of the Italian version of the Chronic Liver Disease Questionnaire (CLDQ-I) for the assessment of health-related quality of life. Digestive and Liver Disease 2005; 37: 850-60. 
14. Ferrer M, Cordoba J, Garin O, et al. Validity of the Spanish version of the chronic liver disease questionnaire (CLDQ) as a standard outcome for quality of life assessment. Liver Transplant 2006; 12: 95-104.

15. Häuser W, Schnur M, Steder-Neukamm U, Muthny FA, Grandt. Validation of the German version of the Chronic Liver Disease Questionnaire. European Journal of Gastroenterology and Hepatology 2004; 16: 599-606.

16. Assessing health status and quality-of-life instruments: attributes and review criteria. Quality of Life Research 2002; 11: 193-205.

17. Linguistic Validation Process [Internet]. Institute, MAPI
Research. 2002 [cited 2013 Jan 5]. Available from: http:// www.mapi-research-inst.com/lvprocess.asp

18. Skevington SM, Lotfy M, O'Connell KA. The World Health Organization's WHOQOL-BREF quality of life assessment: Psychometric properties and results of the international field trial. A Report from the WHOQOL Group. Quality of Life Research 2004; 13: 299-310.

19. Kumarapeli K, Seneviratne R, Wijeyaratne C. Validation of WHOQOL-BREF to measure quality of life among women with polycystic ovary syndrome (PCOS). Journal of College of Community Physicians of Sri Lanka 2006; 11: $1-10$.

\title{
The development and validation of an instrument to assess caregiver burden in cerebral palsy: Caregiver Difficulties Scale
}

\author{
C J Wijesinghe ${ }^{1}$, P Fonseka ${ }^{2}$, C G Hewage ${ }^{3}$
}

(Index words: caregiver burden, cerebral palsy, Sri Lanka, instrument, development)

\begin{abstract}
Objectives To develop a valid and reliable instrument to assess caregiver burden in caregivers of children with cerebral palsy (CP).

Methods A self-administered, multidimensional instrument - Caregiver Difficulties Scale (CDS) - was developed using a combined qualitative-quantitative approach. Items for the preliminary draft were selected from existing caregiver assessment instruments by consensus of experts or key informant interviews with caregivers and service providers of children with CP. Standard item reduction techniques based on responses of 50 caregivers were used to develop the 25 item final draft of CDS. Multidimensionality of CDS was established by exploratory factor analysis, using responses of 125 caregivers. Construct validity of CDS was confirmed by assessing correlations between CDS score and two other constructs: 'Caregiver quality of life' and 'Severity of disease in the care recipient' in a sample of 90 caregivers. Internal consistency and reliability of CDS were assessed using Cronbach's alpha and testretest reliability.
\end{abstract}

Results A new instrument (CDS) was developed with four subscales measuring caregiver's concerns for child, impact on self, support for caregiving and social and economic strain. Face validity, content validity and consensual validity of CDS was established through the process of item generation. Caregiver quality of life and severity of disease in care recipient demonstrated significant moderate to high correlations $(r \geq 0.3)$ with scores of CDS, confirming construct validity. Both internal consistency and reliability of CDS were satisfactory.

Conclusions CDS is a valid and reliable instrument to assess caregiver burden among caregivers of children with cerebral palsy in Sri Lanka.

Ceylon Medical Journal 2013; 58: 162-7

\section{Introduction}

Provision of care for children with disabilities is a daunting task due to physical and emotional demands of caregiving, changes in the social life of caregiver, economic consequences of caregiving etc. Kasuya and collegues define caregiver burden as 'a multidimensional response to physical, psychological, emotional, social and financial stressors associated with caregiving experience' [1]. Burden of caregiving can adversely affect physical and mental health of caregivers, leading to poor quality of care and unmet patient needs [1]. These effects are even more pronounced on caregivers in developing countries where basic facilities for the disabled are limited and respite

Departments of ${ }^{1}$ Community Medicine and ${ }^{2}$ Psychiatry, Faculty of Medicine, University of Ruhuna, ${ }^{3}$ Department of Community Medicine, Faculty of Medical Sciences, University of Sri Jayawardenapura, Sri Lanka.

Correspondence: CJW, e-mail:<cjw@med.ruh.ac.lk>. Received 5 June 2013 and revised version accepted 3 October 2013. Competing interests: none declared. 